\title{
Endoscopic transpapillary gallbladder stenting to prevent acute cholecystitis in patients receiving fully covered self-expandable metal stents for benign biliary stricture
}

\section{(ㄷ)(우우}

\author{
Authors \\ Morgan Wong, Sergio A. Sánchez-Luna, Tarun Rustagi
}

Institution

Division of Gastroenterology and Hepatology, Department of Internal Medicine, University of New Mexico, New Mexico, United States

submitted 27.12.2020

accepted after revision 31.3.2021

\section{Bibliography}

Endosc Int Open 2021; 09: E1386-E1390

DOI 10.1055/a-1500-8028

ISSN 2364-3722

(c) 2021. The Author(s).

This is an open access article published by Thieme under the terms of the Creative Commons Attribution-NonDerivative-NonCommercial License, permitting copying and reproduction so long as the original work is given appropriate credit. Contents may not be used for commercial purposes, or adapted, remixed, transformed or built upon. (https://creativecommons.org/licenses/by-nc-nd/4.0/)

Georg Thieme Verlag KG, Rüdigerstraße 14,

70469 Stuttgart, Germany

\section{Corresponding author}

Tarun Rustagi, MD, Division of Gastroenterology and Hepatology, Department of Internal Medicine, University of New Mexico, MSC10 5550, 1 University of New Mexico, Albuquerque NM 87131, United States

Fax: +1- 505-272-9751

tarunrustagi06@gmail.com

\section{ABSTRACT}

Background and study aims Fully covered self-expanding metal stents (FCSEMS) are being increasingly used for benign biliary strictures (BBS); however, they are associated with risk of acute cholecystitis. Prophylactic endoscopic transpapillary gallbladder stenting (ETPGBS) can facilitate continuous gallbladder drainage and prevent acute cholecystitis from occlusion of cystic duct orifice by the FCSEMS. The aim of this study was to assess the technical feasibility, efficacy, and safety of ETPGBS to prevent acute cholecystitis in patients receiving FCSEMS for BBS.

Patients and methods This was a retrospective analysis of a prospectively collected database at a single center of all patients who underwent prophylactic ETPGBS with FCSEMS for BBS between December 1, 2016 and November 30, 2020.

Results A total of 71 ETPGBS were placed during the study period. Sixteen patients (mean age: $66.4 \pm 19.8$ years; $81 \%$ male) underwent ETPGBS prior to biliary FCSEMS during the same endoscopic session. FCSEMS were left in place (stent dwell time) for a median of 173 days (range: 69-473; mean 196 \pm 121 ) with resolution of BBS and successful removal of ETPGBS and FCSEMS in 12 patients. There was significant improvement in total bilirubin level $(5.25 \pm 5.53$ vs $0.94 \pm 0.85 \mathrm{gm} / \mathrm{dL} ; P=0.008)$. No episodes of acute cholecystitis or any other post-procedural complications were noted during the median follow-up of 337 days (range: 150-856; mean $394 \pm 236$ ).

Conclusions ETPGBS prevented stent-related acute cholecystitis with continued efficacy of FCSEMS for BBS.

\section{Introduction}

Benign biliary strictures (BBS) are a not uncommon occurrence that typically arises from inflammation due to trauma, postoperative injury, anastomotic stricture, chronic pancreatitis, primary sclerosing cholangitis, and gallstone-related strictures [1]. Rates of BBS vary from approximately $0.5 \%$ in cholecystectomy patients to as high as $4 \%$ to $9 \%$ following liver transplantation [2]. Clinical presentation of BBS may be broad and includes a subclinical rise in liver function tests to complete biliary obstruction including jaundice, cholangitis, and chronic cholestasis with secondary biliary cirrhosis [1].

Treatment of BBS includes endoscopic therapy, a percutaneous approach, and surgery. Endoscopic therapy is considered first-line treatment and has evolved over time and includes dilation with biliary balloons, placement of multiple plastic stents (MPS) and fully-covered self-expanding metal stents (FCSEMS) [1]. Although efficacious, placement of plastic biliary stents is 
technically demanding and requires exchange every 3 to 4 months for approximately 1 year $[1,3,4]$.

With recent advances in endoscopy, treatment of BBS with FCSEMS has become an increasingly common practice due the reduced number of endoscopic sessions required and the efficacy in resolution of benign strictures $[1,5,6]$. A recent metaanalysis demonstrated a comparable rate of stricture resolution with a significant decrease in endoscopic retrograde cholangiopancreatography (ERCP) sessions from two sessions in patients receiving FCSEMS in comparison to five in those receiving MPS as a primary treatment modality [7]. The duration of FCSEMS ranges from 3 to 6 months when compared to $>12$ months for MPS and may ultimately result in an increase in the rates of FCSEMS usage.

Although FCSEMS is effective in treatment of BBS, there are multiple associated adverse events (AEs) with usage of FCSEMS including pancreatitis, proximal and distal stent migration, acute cholecystitis, and stent occlusion. Stent-related acute cholecystitis has been reported in up to $12 \%$ of patients receiving FCSEMS [8]. Placement of a prophylactic endoscopic transpapillary gallbladder stent (ETPGBS) prior to placement of a biliary FCSEMS could prevent occlusion of the cystic duct orifice from FCSEMS, allowing for continuous gallbladder drainage, thereby reducing the incidence of post-procedural acute cholecystitis. The aim of this study was to assess the technical feasibility, efficacy, and safety of ETPGBS placement to prevent acute cholecystitis in patients receiving FCSEMS for BBS.

\section{Patients and methods}

\section{Study design and population}

A retrospective analysis of a prospectively collected database at a single tertiary-care referral center of all patients who underwent ETPGBS placement between December 1, 2016 and November 30, 2020 was performed. Patients who underwent ETPGBS prior to placement of FCSEMS for BBS were identified and included in this study. Only patients with an intact gallbladder with cystic duct orifice within or near the bile duct stricture and likely to be covered by the FCSEMS underwent placement of prophylactic ETPGBS and were included in this study. This study was approved by the Institutional Review Board of the University of New Mexico Health Sciences Center.

Technical success was defined as successful placement of both an ETBGB stent and biliary FCSEMS, based on fluoroscopic and endoscopic interpretation. Clinical success was defined as clinical resolution of biliary stricture on both fluoroscopic and endoscopic interpretation, and/or a decrease in serum bilirubin of $>50 \%$ within 2 weeks of FCSEMS placement.

\section{Stent procedure}

A single operator performed all procedures. During ERCP, selective biliary cannulation and a cholangiogram were obtained in the standard fashion to define the biliary stricture. All patients underwent biliary sphincterotomy at index ERCP. Cytology brushings and/or intraductal biopsies were obtained from the stricture to exclude underlying malignancy. The strictures were dilated using a $4-\mathrm{mm}$ or $6-\mathrm{mm}$ biliary dilating balloon catheter (Fusion Titan, Cook Medical, Bloomington, Indiana, United States) in selected cases to facilitate placement of stents across the high-grade stricture. Prior to placement of the FCSEMS, the cystic duct was selectively cannulated using a 0.035-inch angled Glidewire (Terumo Medical Corporation, Somerset, New Jersey, United States). Additional guidewires [0.025-inch angled Visiglide2 (Olympus Medical, Center Valley, Pennsylvania, United States), 0.018-inch angled gold-tipped Terumo guidewire (Terumo Medical Corporation, Somerset, New Jersey, United States) and catheters (rotatable Truetome sphincterotome, Boston Scientific, Marlborough, Massachusetts, United States), 3-4-5 Fr ultra-tapered tip catheter (Contour ERCP Cannula, Boston Scientific, Marlborough, Massachusetts, United States), and 9- to 12-mm Extractor Pro XL stone extraction balloon (Boston Scientific, Marlborough, Massachusetts, United States) were used as needed to achieve successful cannulation in difficult cases. The guidewire was advanced into the gallbladder and allowed to coil within the gallbladder lumen under fluoroscopic guidance. Next, a transpapillary $7 \mathrm{Fr} \times 22 \mathrm{~cm}$ hydrophilic coated, double-pigtail, soft plastic stent (Polaris; Boston Scientific, Marlborough, Massachusetts, United States) was placed across the cystic duct into the gallbladder with the proximal end of the pigtail placed in the gallbladder lumen and distal end in the duodenal lumen.

Following successful placement of the gallbaldder stent, the common bile duct was again cannulated using the 0.035 -inch angled guidewire and the extraction balloon catheter. A transpapillary FCSEMS (ranging from $10 \mathrm{~mm} \times 40-100 \mathrm{~mm}$ ) (WallFlex Biliary RX Stent; Boston Scientific, Marlborough, Massachusetts, United States or GORE VIABIL Biliary Endoprosthesis; Conmed, Utica, New York, United States) was placed across the biliary stricture under endoscopic and fluoroscopic guidance.

\section{Results}

A total of 71 ETPGBS were placed during the study period December 1, 2016 to November 30, 2020 for endoscopic management of acute cholecystitis or as prophylactic ETPGBS prior to biliary FCSEMS for management of BBS. Selective cystic duct and gallbladder wire cannulation was achieved followed by successful placement of ETPGBS in all patients with a technical success rate of $100 \%$.

Sixteen patients with BBS (mean age: $66.4 \pm 19.8$ years; $81 \%$ male) underwent ETPGBS prior to biliary FCSEMS during the same endoscopic session ( $\mathbf{F i g . 1}$ ). All patients had MRCP, computed tomography, or endoscopic ultrasound prior to ERCP. The location of the cystic duct orifice was evaluated on these imaging studies and confirmed on ERCP. The cystic duct orifice was located within the proximal portion of the bile duct stricture in three patients and just proximal (within $1 \mathrm{~cm}$ of the stricture) in 13 patients, such that the cystic duct orifice would be covered by placement of an adequate length of FCSEMS in all 16 patients. One patient was subsequently diagnosed with a malignant biliary stricture and was excluded from further analysis. The etiology of BBS in the remaining 15 patients included chronic pancreatitis ( $n=9$ patients), acute necrotizing pancrea- 

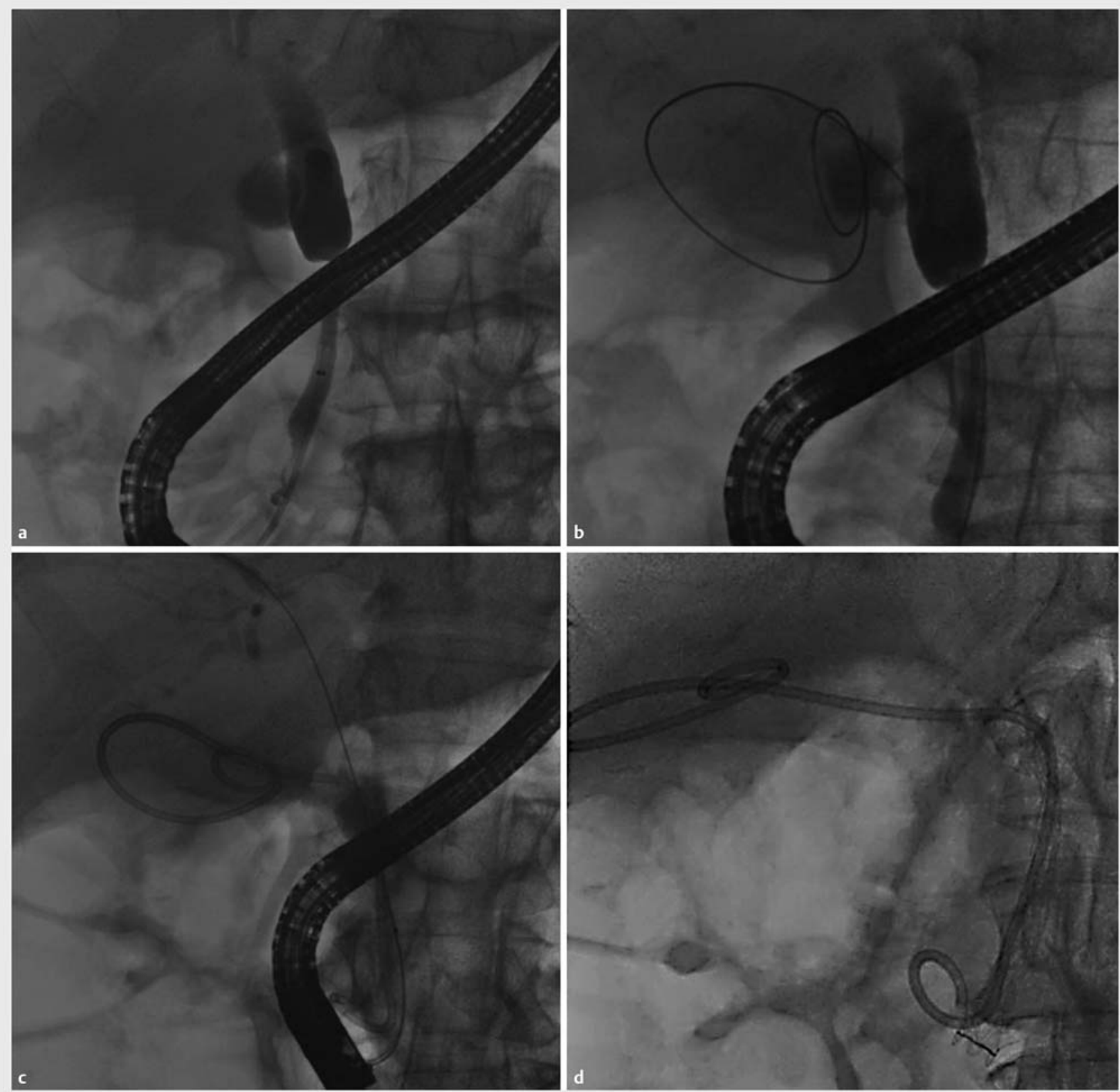

- Fig. 1 Cholangiogram showing a a biliary stricture followed by $\mathbf{b}$ selective wire cannulation of the cystic duct and coiling of the guidewire in the gallbladder. $\mathbf{c}$ A transpapillary double-pigtail plastic gallbladder stent was placed, followed by $\mathbf{d}$ biliary FCSEMS placement for management of a biliary stricture.

titis $(n=3)$, choledocholithiais-related $(n=1)$, pancreatic head cyst $(n=1)$, and idiopathic stricture $(n=1)$.

Technical success was achieved in all patients (100\%). In all cases, a 10-mm-diameter FCSEMS (length ranging from $40 \mathrm{~mm}$ to $100 \mathrm{~mm}$; $60 \mathrm{~mm}$ being the most common) was placed across the common bile duct stricture. All patients included this study had FCSEMS covering the cystic duct orifice. There was significant improvement in total bilirubin level (pre-procedural $5.25 \pm$ 5.53 vs post-procedural $0.94 \pm 0.85 \mathrm{gm} / \mathrm{dL}$ with mean reduction of $4.31 \pm 5.57$ (95\% Cl: 1.23 to $7.38 ; P=0.0079)$.
FCSEMS were left in place (stent dwell time) for a median of 173 days (range: 69-473; mean 196 \pm 121 ) with resolution of BBS and successful removal of ETPGBS and FCSEMS in 12 patients. Three patients died due to unrelated events (complications of decompensated cirrhosis in 1, cardiac etiology in 2).

AEs included obstruction of FCSEMS in one patient requiring repeat ERCP with balloon sweeps with clearing of the stent. Another patient had spontaneous FCSEMS migration 4 months after initial placement with resolution of stricture. No episodes of acute cholecystitis or any other immediate or late AEs related 
to ETPGBS were noted during the median follow-up of 337 (range: 150-856; mean 394 \pm 236 ) days.

\section{Discussion}

Treatment of BBS with FCSEMS has become an increasingly common alternative therapy to MPS. FCSEMS have been shown to have similar or higher efficacy compared to MPS in achieving resolution of BBSs with fewer ERCP procedures [5,6]. This translates into fewer procedural and post-procedural complications and overall cost reduction as fewer ERCPs are required. However, stent-related acute cholecystitis may develop in $3 \%$ to $12 \%$ of patients treated with FCSEMS and may lead to unplanned FCSEMS removal in such patients [8-12].

ETPGBS has been reported for management of stent-related acute cholecystitis developing after placement of FCSEMS for biliary strictures $[13,14]$. In such cases, the previously placed FCSEMS is removed followed by ETPGBS and placement of a new FCSEMS across the biliary stricture. Our study demonstrates the value of utilizing ETPGBS at the time of FCSEMS placement to decrease the post-procedural risk of acute cholecystitis in patients with BBS treated with FCSEMS.

Post-cholecystectomy patients with BBS are likely to receive FCSEMS as there is no risk of acute cholecystitis in such patients. However, FCSEMS use in patients with an intact gallbladder has to be evaluated to avoid risk of cholecystitis. The risk of acute cholecystitis reported in studies using FCSEMS for BBS is low, most likely due to selection bias. Patients in whom placement of a FCSEMS would overlap the cystic duct in the setting of an intact gallbladder are typically excluded from the studies to avoid the potential risk for stent-induced acute cholecystitis [6]. Many patients do not receive an FCSEMS if the length required for BBS is likely to cover the cystic duct orifice or if cystic duct take off cannot be ascertained; in these cases, MPS is pursued. Patients who receive an FCSEMS for BBS are carefully selected; therefore, the true risk of acute cholecystitis in them is unknown.

ETGBS placement prior to FCSEMS in our study was shown to be an effective treatment for BBS with no increase in procedural or post-procedural complications. ETPGBS prevented stentrelated acute cholecystitis with continued efficacy of FCSEMS. This technique could also be used to prevent cholecystitis in patients who have indications other than BBS for which FCSEMS are indicated or preferred, such as bile duct injury or perforation, bile leak, and post-sphincterotomy bleeding. Most of these patients, however, require FCSEMS for a shorter time (with likely smaller risk of acute cholecystitis) and it may be technically challenging and time-consuming to perform ETPGBS in such urgent cases. In addition, this technique could also be considered in patients with malignant biliary strictures and intact gallbladder to prevent acute cholecystitis with the advantages of FCSEMS over uncovered SEMS.

We used a specific hydrophilic-coated double-pigtail soft plastic stent (Polaris; originally designed to be used as a ureteral stent). This stent comes only in $20-\mathrm{cm}$ and $22-\mathrm{cm}$ lengths. Shorter stents would likely be better in some patients. However, given the very soft material of this stent, the stent easily coils within the gallbladder lumen and the distal pigtail easily resides within the distal duodenum without any AEs noted in any of the 71 patients who underwent ETPGBS using this stent.

ETGBS adds to the procedure time, which is a disadvantage of this technique. However, the procedure (once biliary cannulation and sphincterotomy have been achieved) does not add significant time, particularly considering the benefits of this procedure, which facilitates placement of FCSEMS with reduced risk of acute cholecystitis. In addition, overall procedure time is saved as the number of ERCPs required with FCSEMS is less compared to sequential MPS placement requiring repeated ERCPs, with each adding to the total procedural time.

Our study has a few limitations. First, this was a retrospective, single-center, single-operator study with a relatively small sample size, which may reflect the low incidence of BBS. Not only the incidence of BBS is low. Many patients with BBS had cholecystectomy and did not need ETPGBS; therefore, they were not eligible for inclusion in our study. Only patients with BBS with an intact gallbladder with cystic duct orifice identified within the stricture or just above the stricture such that an adequate-length FCSEMS would cover the cystic duct orifice were eligible for the study. This significantly reduced the number of patients. In addition, the risks and benefits of this technique need to be further evaluated, given that the exact incidence of acute cholecystitis after FCSEMS placement for BBS is unknown and probably low, as discussed earlier. Furthermore, selection of the type of FCSEMS type was at the discretion of the endoscopist, not using a pre-established protocol. Finally, our study was conducted in a high-volume center (>1000 ERCPs/year) with extensive experience and expertise in performing ETPGBS with high success rates and the results of our study may not be applicable to lower-volume centers with less experience in complex ERCP techniques. However, to the best of our knowledge, this is the first study to evaluate the technical feasibility, efficacy, and safety of prophylactic ETPGBS in preventing acute cholecystitis in patients receiving FCSEMS for BBS.

\section{Conclusions}

In conclusion, treatment of BBS with FCSEMS has become an increasingly common alternative therapy to plastic biliary stents. In our study, ETPGBS placement prior to FCSEMS was an effective treatment for BBS with no increase in intraoperative or post-procedure complications. ETPGBS prevented post-procedure acute cholecystitis with continued efficacy of FCSEMS. Future larger, prospective, ideally randomized controlled studies are required to validate our findings prior to more widespread clinical implementation.

\section{Competing interests}

Dr. Rustagi is a consultant for Boston Scientific. 


\section{References}

[1] Rustagi T, Jamidar PA. Endoscopic management of benign biliary strictures. Curr Gastroenterol Rep 2015; 17: 422

[2] Ma MX, Jayasekeran V, Chong AK. Benign biliary strictures: prevalence, impact, and management strategies. Clin Exp Gastroenterol 2019; 12: 83-92

[3] Costamagna G, Shah SK, Tringali A. Current management of postoperative complications and benign biliary strictures. Gastrointest Endosc Clin N Am 2003; 13: 635-648

[4] Costamagna G, Bulajic M, Tringali A et al. Multiple stenting of refractory pancreatic duct strictures in severe chronic pancreatitis: longterm results. Endoscopy 2006; 38: 254-259

[5] Zheng X, Wu J, Sun B et al. Clinical outcome of endoscopic covered metal stenting for resolution of benign biliary stricture: Systematic review and meta-analysis. Dig Endosc 2017; 29: 198-210

[6] Cote GA, Slivka A, Tarnasky P et al. Effect of covered metallic stents compared with plastic stents on benign biliary stricture resolution: a randomized clinical trial. JAMA 2016; 315: 1250-1257

[7] Khan MA, Baron TH, Kamal F et al. Efficacy of self-expandable metal stents in management of benign biliary strictures and comparison with multiple plastic stents: a meta-analysis. Endoscopy 2017; 49: 682-694
[8] Pausawasadi N, Soontornmanokul T, Rerknimitr R. Role of fully covered self-expandable metal stent for treatment of benign biliary strictures and bile leaks. Korean J Radiol 2012; 13: S67-73

[9] Deviere J, Nageshwar Reddy D et al. Successful management of benign biliary strictures with fully covered self-expanding metal stents. Gastroenterology 2014; 147: 385-395 quiz e315

[10] Lakhtakia S, Reddy N, Dolak W et al. Long-term outcomes after temporary placement of a self-expanding fully covered metal stent for benign biliary strictures secondary to chronic pancreatitis. Gastrointest Endosc 2020; 91: 361-369 e363

[11] Jang S, Stevens T, Parsi M et al. Association of covered metallic stents with cholecystitis and stent migration in malignant biliary stricture. Gastrointest Endosc 2018; 87: 1061-1070

[12] Bezzi M, Zolovkins A, Cantisani V et al. New ePTFE/FEP-covered stent in the palliative treatment of malignant biliary obstruction. J Vasc Interv Radiol 2002; 13: 581-589

[13] Kawakubo K, Isayama H, Sasahira $\mathrm{N}$ et al. Endoscopic transpapillary gallbladder drainage with replacement of a covered self-expandable metal stent. World J Gastrointest Endosc 2011; 3: 46-48

[14] Nakahara K, Morita R, Michikawa Y et al. Endoscopic Transpapillary gallbladder drainage for acute cholecystitis after biliary self-expandable metal stent placement. Surg Laparosc Endosc Percutan Tech 2020; 30: 416-423

\section{CORRECTION}

Endoscopic transpapillary gallbladder stenting to prevent acute cholecystitis in patients receiving fully covered self-expandable metal stents for benign biliary stricture

Morgan Wong, Sergio A. Sánchez-Luna, Tarun Rustagi Endoscopy International Open 2021; 09: E1386-E1390.

DOI: $10.1055 / a-1500-8028$

The title of the article was corrected. Correct is: Endoscopic transpapillary gallbladder stenting to prevent acute cholecystitis in patients receiving fully covered self-expandable metal stents for benign biliary stricture. This was corrected in the online version on September 6, 2021. 